\title{
Advanced PID Controller Synthesis using Multiscale Control Scheme
}

\author{
Bejay Ugon ${ }^{\mathrm{a}, *}$, Jobrun Nandong ${ }^{\mathrm{a}}$, and Zhuquan Zang ${ }^{\mathrm{b}}$ \\ ${ }^{a}$ Department of Chemical Engineering, Curtin University, 98009 Miri, Sarawak, Malaysia \\ ${ }^{\mathrm{b}}$ Department of Electrical and Computer Engineering, Curtin University, 98009 Miri, Sarawak, Malaysia \\ \{bejay@curtin.edu.my, jobrun.n@curtin.edu.my, zqzang@curtin.edu.my\}
}

\begin{abstract}
The PID controller has been widely applied in industries since many decades ago despite the advancement in many advanced control techniques. Process models such as the First-Order plus Deadtime (FOPDT) has often been used to design or tune the PID controller. A large number of PID controller tuning formulas have been established since the wellknown Ziegler-Nichols formula introduced in the 1940s. In this paper, we present a new approach based on the Multi-scale Control scheme to constructing a PID controller tuning formula which is applicable to the FOPDT model. The effectiveness of the proposed PID tuning formula is compared with some of the best PID tuning formulas reported in the literature.
\end{abstract}

Keywords-PID Tuning; Time-Delay; Multiscale Control

\section{INTRODUCTION}

In process industry, the presence of time delay or deadtime often causes poor control performance or even closed-loop instability. In fact, the presence of deadtime has been recognized as one of the limiting factors for the closed-loop performance [1]. Interestingly, the model which incorporates deadtime, i.e., the First-Order plus Deadtime (FOPDT) model has been used rather extensively in the PID controller design, i.e., PID controller tuning formulas or rules. Since the wellknown Ziegler-Nichols PID tuning formula introduced in the 1940s, a large number of PID tuning formulas based on the FOPDT model have been developed; see the summary report in [2].

In this brief paper, we shall demonstrate a new approach to devising an advanced PID controller augmented with a filter; this PID formula is derived based on the principle of the Multiscale Control (MSC) scheme recently introduced by Nandong and Zang [3].

The rest of this paper is organized as follows. Section II provides a brief overview of the MSC scheme and a PID tuning formula derivation based on the FOPDT model. Section III demonstrates the effectiveness of the proposed PID tuning formula using two case studies: Steam Superheater and Cement Cooler Grate systems. Finally, Section IV highlights some conclusions and future works.

\section{Multi-ScAle CONTROL SCHEME}

\section{A. Multi-scale Plant Decomposition}

The details about the Multi-scale Control (MSC) scheme can be found in [3]. Here, we only provide a brief overview of the MSC scheme. The principle of the MSC scheme is first to decompose a given plant into a sum of basic factors or modes with distinct speed responses. For a general case, consider a rational transfer function $P$ which can be decomposed into a sum of $n+1$ basic factors or modes as follows:

$P(s)=\frac{N(s)}{D(s)}=P_{0}(s)+P_{1}(s)+P_{2}(s)+\ldots+P_{n}(s)$

Here, $P_{i}, \forall i \in\{0,1,2, \ldots, n\}$ is the plant factor, which is either first or second order system with real coefficients. The dynamic of $P_{i}$ is slower than that of $P_{i+1}$ for $i=0,1,2, \ldots, n ; P_{0}$ is called the outermost factor while $P_{i}, \forall i \in\{1,2,3 \ldots, n\}$ the innerlayer factor. Notice that this general case results in a $n+1$ layer MSC scheme. In real application, we might just need a 2or 3-layer MSC scheme. In this present paper, we demonstrate the application of the 2-layer MSC scheme to constructing a PID tuning formula based on the First-Order plus Deadtime (FOPDT) model.

\section{B. Realization of the 2-Layer Multi-scale Control Scheme}

The block diagram of a 2-layer multi-scale control (MSC) scheme is shown in Figure 1; i.e., a given plant $P$ can be decomposed into 2 modes ( $\left.P=P_{0}+P_{1}\right)$. Here, $W_{i}$ is called the multi-scale predictors; $K_{i}$ the multi-scale sub-controllers; $P_{c}$ the augmented overall plant transfer function. The outermost sub-controller $\left(K_{0}\right)$ corresponds to the factor with the slowest dynamic $\left(P_{0}\right)$ and $K_{1}$ corresponds to the fastest dynamic $\left(P_{2}\right)$.

In Figure 1, the closed-loop transfer function for the inner layer is given by:

$$
G_{1}(s)=\frac{K_{1}(s)}{1+K_{1}(s) W_{1}(s)}
$$


The augmented overall plant transfer function is expressed as

$$
P_{c}(s)=G_{1}(s) P(s)
$$

The overall MSC controller is then given by

$$
K_{m s c}(s)=G_{1}(s) K_{0}(s)
$$
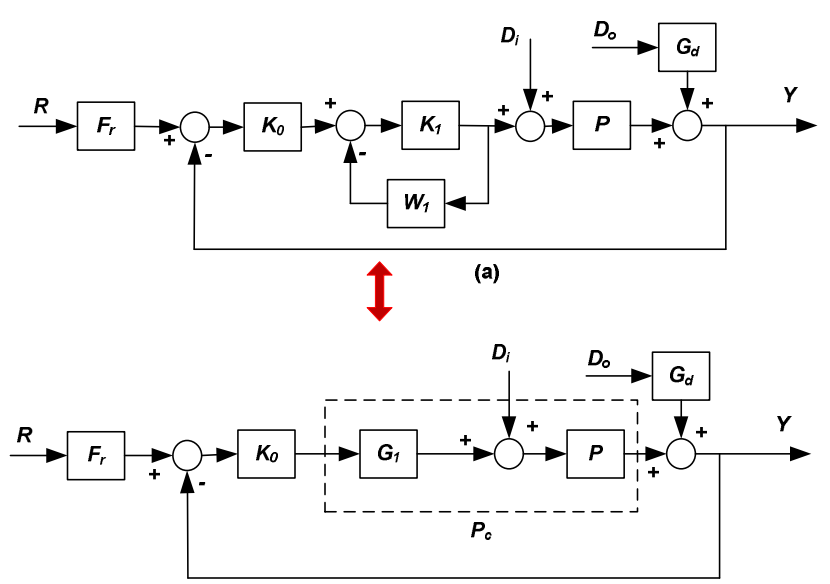

(b)

Figure 1. Block diagram of 2-layer multi-scale control scheme: (a) full 2loop, (b) reduced single-loop [3]

\section{Derivation of PID Tuning Formula}

Let us consider a process which can represented by the First-Order Plus Deadtime (FOPDT) model as follows

$$
P(s)=\frac{K_{p} e^{-\theta s}}{\tau s+1}
$$

where $K_{p}, \tau, \theta$ denote the process gain, time constant and deadtime respectively.

By using 1/1 Padé formula to approximate the delay component in (4) gives

$$
P(s)=\frac{K_{p}(1-\alpha s)}{(\tau s+1)(1+\alpha s)}
$$

where $\alpha=0.5 \theta$.

Assuming that $\tau>\alpha$, and after applying partial fraction expansion to (5), we obtain 2 modes as follows

$$
P(s)=\frac{A}{\tau+1}+\frac{B}{\alpha s+1}
$$

$$
A=K_{p} \frac{\tau+\alpha}{\tau-\alpha}
$$

$$
B=\frac{2 \alpha K_{p}}{\alpha-\tau}
$$

The multi-scale predictor is chosen as the inner mode, i.e.

$$
W(s)=\frac{B}{\alpha s+1}
$$

Assuming P-only controller with gain $K_{c 1}$ is used in the inner-loop, the following closed-loop setpoint transfer function is obtained

$$
H_{r 1}(s)=G_{1}(s)=\frac{K_{c 1}}{1+K_{c 1} W(s)}
$$

The equation (10) can be simplified to

$$
G_{1}(s)=\frac{K_{c 1}^{o}(\alpha s+1)}{\tau_{c 1} s+1}
$$

where the overall gain and closed-loop time constant are given respectively by

$$
\begin{gathered}
K^{o}{ }_{c 1}=\frac{K_{c 1}}{1+K_{c 1} B} \\
\tau_{c 1}=\frac{\alpha}{1+K_{c 1} B}
\end{gathered}
$$

Now, consider the ratio of open-loop to closed-loop time constant as given by

$$
\lambda_{1}=\frac{\alpha}{\tau_{c 1}}
$$

From (13) and (14), $K_{c 1}$ can be expressed as follows

$$
K_{c 1}=\frac{\lambda_{1}-1}{B}
$$

Note that, the greater the value of $\lambda_{1}$, the faster is the closed-loop response, i.e., more aggressive controller action. 
Next, let us consider that a PI controller is chosen to control the outermost mode, i.e.

$$
K_{0}(s)=K_{c 0}\left(1+\frac{1}{\tau_{I 0} s}\right)
$$

where $K_{c 0}$ and $\tau_{I 0}$ indicate the sub-controller gain and reset time respectively.

To determine $K_{c 0}$ assume first that the outermost is controlled using a P-only controller. Then it can be determined in the same way as the $\mathrm{P}$ controller for the inner mode, i.e.

$$
K_{c 0}=\frac{\lambda_{0}-1}{A}
$$

where $\lambda_{0}$ is the ratio of the open-loop to closed-loop time constant, i.e., defined in the same way as (14).

It follows that, the reset time for the PI controller is set based on a fraction of the open-loop time constant for the outermost mode as follows

$$
\tau_{I 0}=\gamma \tau, \quad 0.3 \leq \gamma \leq 1.2
$$

The overall MSC controller (3) can now be arranged in the form of

$$
K_{m s c}(s)=\frac{K_{c 0} K_{c 1}^{o}}{\tau_{I 0}} S\left(K_{c 1}\right)\left(\frac{\tau_{I 0} s+1}{s}\right)\left(\frac{\alpha s+1}{\tau_{c 1} s+1}\right)
$$

Here, $S\left(K_{c 1}\right)$ denotes the sign of controller gain $K_{c 1}$, which needs to be included in (19) to get the correct sign for the overall controller gain.

It can be easily shown that the overall MSC controller (18) can be expressed as a practical PID controller augmented with a filter given by

$$
K_{m s c}(s)=K_{c}\left(1+\frac{1}{\tau_{I} s}+\tau_{D^{S}}\right)\left(\frac{1}{\tau_{f} s+1}\right)
$$

The equivalent MSC-PID tuning parameters $K_{c}, \tau_{I}$ and $\tau_{D}$ are expressed as

$$
\begin{gathered}
K_{c}=\frac{K_{c 0} K_{c 1}^{o} S\left(K_{c 1}\right)\left(\tau_{I 0}+\alpha\right)}{\tau_{I 0}} \\
\tau_{I}=\tau_{I 0}+\alpha
\end{gathered}
$$

$$
\tau_{D}=\frac{\tau_{I 0} \alpha}{\tau_{I 0}+\alpha}
$$

The parameters above can be further expressed in terms of model and our specification parameters

$$
\begin{gathered}
K_{c}=\left(\frac{\left(\lambda_{0}-1\right)\left(\lambda_{1}-1\right)\left(\gamma \tau+\frac{\theta}{2}\right)\left(\tau-\frac{\theta}{2}\right)\left(\frac{\theta}{2}-\tau\right)}{\left.\gamma \theta \tau K_{p}\left(\tau+\frac{\theta}{2}\right) \lambda_{1}\right)}\right) S\left(K_{c 1}\right) \\
\tau_{I}=\gamma \tau+0.5 \theta \\
\tau_{D}=\frac{\gamma \theta \tau}{2 \gamma \tau+\theta} \\
\tau_{f}=\frac{\theta}{2 \lambda_{1}}
\end{gathered}
$$

\section{Remarks:}

- The controller specifications are $\lambda_{0}, \lambda_{1}$ and $\gamma$ in order to obtain the MSC-PID parameters via (24) (27).

- The $\lambda_{0}$ and $\lambda_{1}$ are adjusted first while keeping $\gamma=1$ until gain margin (GM) approximately $7 \mathrm{~dB}$ is reached. Then, $\gamma$ is slightly adjusted to get a desired final response.

\section{ILLUSTRATIVE EXAMPLES}

\section{Example 1- Steam Superheater}

A linearized model for the Steam Superheater main temperature (i.e., from Loop-pro Control Station, Inc.) is obtained as follows:

$$
P(s)=\frac{-7.16 e^{-27.7 s}}{27.7 s+1}
$$

Additionally, a perturbed model for the Steam Superheater temperature is given by

$$
P_{\Delta}(s)=\frac{-6.19 e^{-35 s}}{(17.5 s+1)^{2}}
$$

Upon approximating the time-delay component in (28) using the 1/1 Padé formula, the nominal model (28) can be written as follows: 


$$
P(s)=\frac{-7.16(1-13.85 s)}{(27.7 s+1)(13.85 s+1)}
$$

Next, we can decompose (30) into two factors using partial fraction expansion:

$$
\left[\begin{array}{l}
P_{0}(s) \\
P_{1}(s)
\end{array}\right]=\left[\begin{array}{c}
\frac{-3}{27.7 s+1} \\
\frac{14.32}{13.85 s+1}
\end{array}\right]
$$

where the multi-scale predictor is chosen as the inner mode

$$
W(s)=\frac{14.32}{13.85 s+1}
$$

For performance comparison, two other PID controller base on FOPDT model are designed with minimum IAE formula of Rovira et al. [4] and Suyama [5] for ideal PID controller given by:

$$
G_{c}=K_{c}\left(1+\frac{1}{T_{i} s}+T_{d} s\right)
$$

Note that, the Rovira et al. [4] PID tuning formula is based on servo control while that of Suyama [5] is based on direct synthesis control.

The tuning parameter for the MSC-PID controller is based on the settings: $\lambda_{0}=3.9, \lambda_{1}=120$ and $\gamma=0.69$, which gives $\mathrm{GM}=9.16 \mathrm{bB}, \mathrm{PM}=60^{\circ}$ and $\mathrm{DM}=43.9$ seconds. Tuning parameter for Rovira et al.[3] give $\mathrm{GM}=6.42, \mathrm{PM}=71.3^{\circ}$ and $\mathrm{DM}=46.3$ seconds and tuning parameter for Suyama[5] give $\mathrm{GM}=7.9 \mathrm{~dB}, \mathrm{PM}=60^{\circ}, \mathrm{DM}=39.9$ seconds. The performances of the 3 different PID controllers are evaluated against 1 unit step change in the setpoint, 0.1 unit in input disturbance and followed by 1 unit in output disturbance. Figures 2 and 3 show the disturbance rejection performances at the nominal condition (28) for the 3 different PID controllers.

For the input type disturbance, it is obvious (Figure 2) that the MSC-PID controller shows superior performance over the PID controllers tuned using Rovira et al. [4] and Suyama [5]. Figures 4 and 5 show the closed-loop responses to input and output disturbances at the perturbed condition given in (29). In term of the IAE value, the MSC-PID provides better performance than the Rovira et al. and Suyama PID controllers.

TABLE I. PID TUNING PARAMETERS FOR STEAM SUPERHEATER.

\begin{tabular}{lllll}
\hline PID Formula & $K_{c}$ & $\tau_{I}$ & $\tau_{D}$ & $\tau_{f}$ \\
\hline Rovira et al.[4] & -0.1522 & 45.4098 & 9.695 & - \\
Suyama [5] & -0.1397 & 27.7 & 13.85 & - \\
Proposed MSC & -0.1155 & 32.963 & 8.0307 & 0.1154 \\
\hline
\end{tabular}

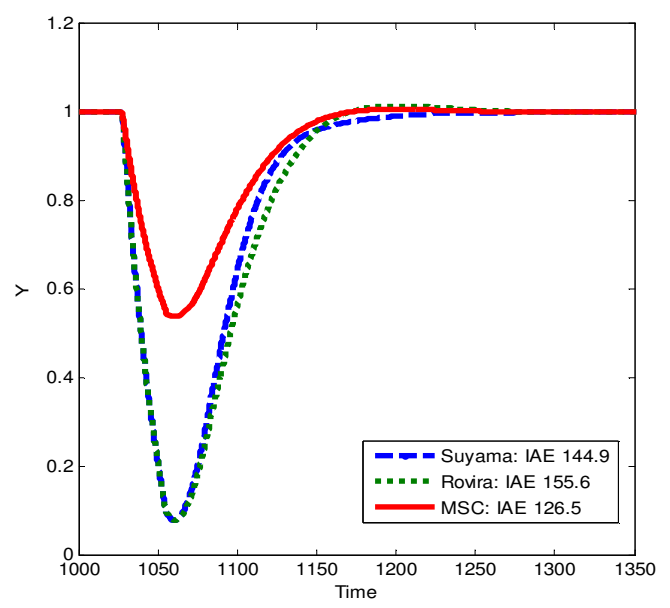

Figure 2. Input disturbance rejection response at nominal condition for the Steam Superheater

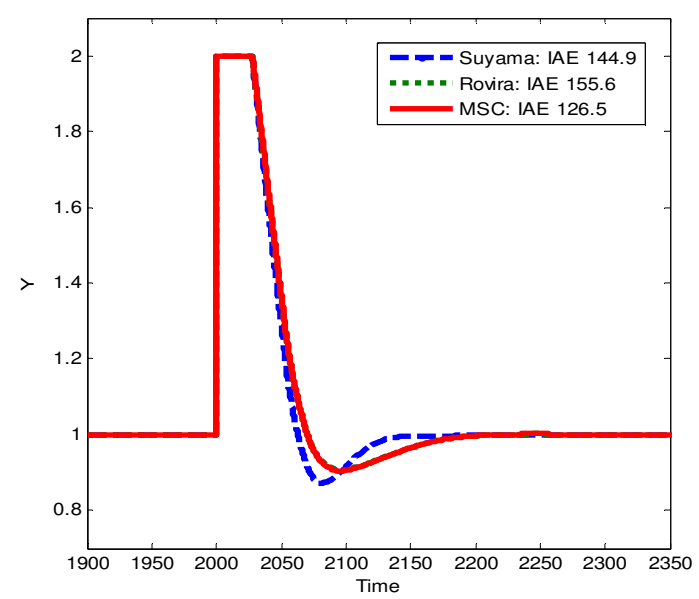

Figure 3. Output disturbance rejection response at nominal condition for the Steam Superheater

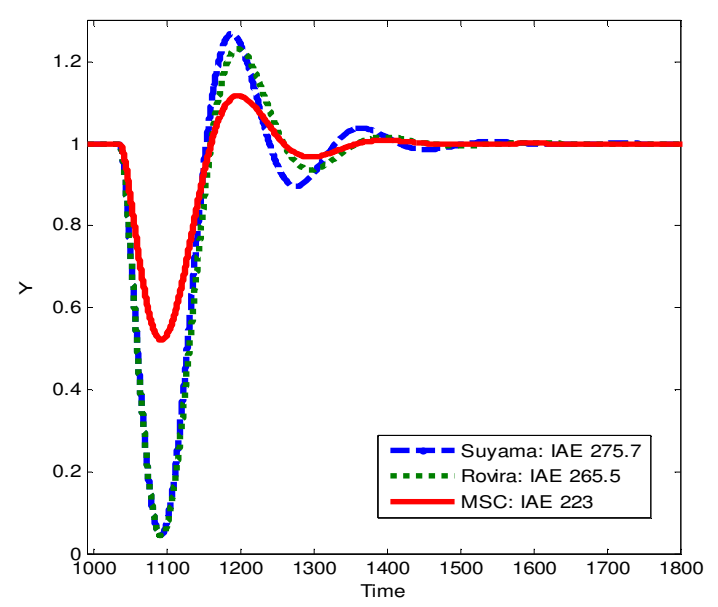

Figure 4. Input disturbance rejection performance at perturbed condition for the Steam Superheater 


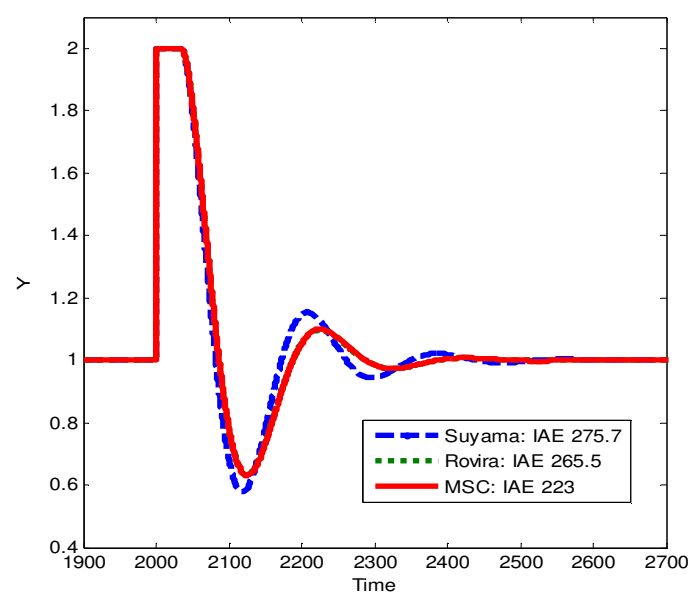

Figure 5. Output disturbance rejection response at perturbed condition for the Steam Superheater.

Overall, in term of the total Integral Absolute Error (IAE) value, the proposed MSC-PID controller gives the best performance both at the nominal and perturbed conditions; the Rovira et al. PID controller gives better performance than the Suyama PID controller at the perturbed condition, and vice versa at the nominal condition.

\section{Example 2 -Cement Cooler Grate}

A linearized model for the Cement Cooler Grate pressure (i.e., from Loop-pro Control Station, Inc.) is given by:

$$
P(s)=\frac{-43.7 e^{-4.78 s}}{6.18 s+1}
$$

A perturbed linearized model is given by

$$
P_{\Delta}(s)=\frac{-42.2 e^{-7.2 s}}{5.1 s+1}
$$

The application of 1/1 Padé formula to (34) yields an approximated model as follows:

$$
P(s)=\frac{-43.7(1-2.39 s)}{(6.18 s+1)(1+2.39 s)}(36)
$$

The application of partial fraction expansion to (36) leads to

$$
P(s)=\frac{2.26}{6.18 s+1}+\frac{55.115}{2.29 s+1}
$$

The multi-scale predictor is chosen as the inner mode

$$
W(s)=\frac{55.115}{2.29 s+1}
$$

For performance comparison, 3 PID controllers based on the FOPDT model (34) are designed using formulas: (a) Witt \& Waggoner [6], (b) Kaya \& Scheib [7], and (c) proposed MSCPID. The PID tuning formulas of Witt \& Waggoner [6] and Kaya \& Scheib [7] are developed for the classical PID controller of the form

$$
G_{c}=K_{c}\left(1+\frac{1}{T_{i} s}\right)\left(\frac{1+T_{d} s}{1+\frac{T_{d} s}{N}}\right)
$$

Note that, the Witt and Waggoner formula [6] is developed based on the process reaction control, while the Kaya and Scheib formula [7] is based on servo control. In both the Witt $\&$ Waggoner and Kaya \& Scheib formulas, $N=10$ is used. For the proposed MSC-PID scheme, we use these settings: $\lambda_{0}=3.8, \lambda_{1}=10$ and $\gamma=0.8$, which lead to $\mathrm{GM}=6.55 \mathrm{~dB}$ and $\mathrm{PM}=56.2^{\circ}, \mathrm{DM}=5.65$ minutes.

TABLE II. PID CONTROLLER PARAMETERS FOR THE CEMENT COLLER GRATE.

\begin{tabular}{lllll}
\hline PID Formula & $K_{c}$ & $\tau_{I}$ & $\tau_{D}$ & $\tau_{f}$ \\
\hline Witt \& Waggoner [6] & -0.0178 & 4.78 & 4.78 & - \\
Kaya \& Scheib [7] & -0.0195 & 4.2954 & 4.149 & - \\
Proposed MSC & -0.0306 & 7.334 & 1.611 & 0.199 \\
\hline
\end{tabular}

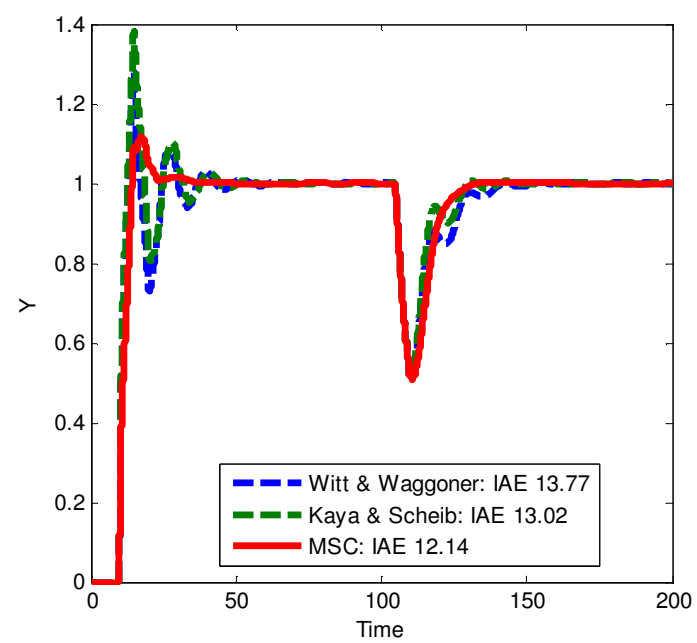

Figure 6. Closed-loop response at norminal condition for the Cement Coller Grate pressure

The comparative performances resulting from the 3 different PID controller tunings at nominal condition for a consecutive unit step change and 0.02 units change in input disturbance are shown in Figure 6. It is clearly shown that the setpoint tracking performance for the MSC-PID controller is better than the other two controllers tuned using Witt \& Waggoner and Kaya \& Scheib formulas. The disturbance rejection performances for the 3 PID controllers are quite the 
same. Overall, the MSC-PID gives the best performance in term of the IAE value, i.e., the smallest IAE value. Figure 7 demonstrate the closed-loop responses for the 3 different PID controllers under a perturbed condition (35). Again, the MSCPID controller provides the best performance.

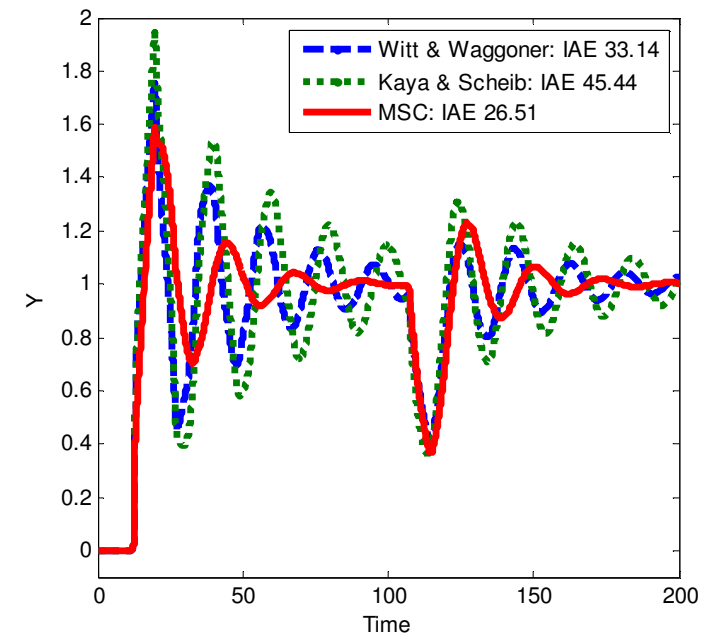

Figure 7. Closed-loop responses at perturbed condition for the Cement Cooler Grate pressure

\section{CONCLUSIONS}

Over the last few decades, a large number of PID tuning formulas based on the First-Order plus Deadtime (FOPDT) model have been developed. In this paper, we have presented a new approach based on the Multi-scale Control (MSC) scheme to constructing a PID tuning formula for a process represented by the FOPDT model. Based on the two industrial processes (Steam Superheater and Cement Cooler Grate), we have shown the superiority of our new PID tuning formula based on the MSC scheme (MSC-PID) over some of the best PID tuning formulas established over the last several decades, e.g., Rovira et al. [4], Suyama [5], Witt \& Waggoner [6] and Kaya \&Scheib [7]. In future works, we will further extend the application of the MSC scheme to constructing a few other PID tuning formulas based on the Second-Order plus Deadtime (SOPDT) and Second-Order Integrating plus Deadtime (SOIPDT) models.

\section{REFERENCES}

[1] K. J. Astrom, "Limitation on control system performance," Eur. J. Control, vol. 6, no. 1, pp. 2-20, 2000.

[2] O. Aidan, "A summary of PI and PID controller tuning rules for processes with time delay. Part 2: PID controller tuning rules," Proceedings of PID: IFAC Workshop on Digital Control, pp. 242-247, Terrasa, Spain April 4-7, 2000.

[3] J. Nandong and Z. Zang, "High-performance multi-scale control scheme for stable, integrating and unstable time-delay processes," vol. 23, pp. 1333-1343.

[4] A.A. Rovira, P.W. Murril and C.L. Smith, "Tuning controller for set point changes," Intruments and Control Systems, pp.67-69, December 1969.

[5] K. Suyama, "A simple design method for sampled-data PID control systems with adequate step responses," Procedings of the International
Conference on Industrial Electronics, Control, Instrumentation and Automation, pp. 1117-1122, 1992.

[6] S.D. Witt and R.C. Waggoner "Tuning parameters for non-PID three mode controllers," Hydrocarbon Processing, pp. 74-78, 1990.

[7] A. Kaya and T.J. Scheib, "Tuning PID controls of different structures," Control Engineering, pp. 62-65, 1988 